\title{
OSCILLATION CRITERION FOR TWO-DIMENSIONAL DYNAMIC SYSTEMS ON TIME SCALES
}

\author{
TAHER S. HASSAN
}

\begin{abstract}
The purpose of this paper is to prove oscillation criterion for dynamic system

$$
u^{\Delta}=p v, \quad v^{\Delta}=-q u^{\sigma},
$$

where $p>0$ and $q$ are rd-continuous functions on a time scale such that $\sup \mathbb{\mathbb { T }}=\infty$ without explicit sign assumptions on $q$ and also without restrictive conditions on the time scale $\mathbb{T}$.
\end{abstract}

\section{Introduction}

We consider the linear dynamic system

$$
u^{\Delta}=p v, \quad v^{\Delta}=-q u^{\sigma},
$$

where $p>0$ and $q$ are rd-continuous functions on a time scale such that sup $\mathbb{T}=\infty$. A solution $(u(t), v(t))$ of system (1.1) is called oscillatory if both $u(t)$ and $v(t)$ are oscillatory functions, and otherwise it will be called nonoscillatory. System (1.1) is called oscillatory if its solutions are oscillatory.

In $[6,1]$, the following oscillation theorem is obtained.

Theorem 1.1. Assume that $q(t) \geq 0$ is rd-continuous function and

$$
\int_{t_{0}}^{\infty} p(t) \Delta t=\infty \quad \text { and } \quad \lim _{t \rightarrow \infty} \frac{\mu(t) p(t)}{\bar{p}(t)}=0 .
$$

If there exists $\lambda \in(0,1)$ such that

$$
\int_{t_{0}}^{\infty} \bar{p}^{\lambda}(t) q(t) \Delta t=\infty
$$

where

$$
\bar{p}(t):=\int_{t_{0}}^{t} p(s) \Delta s
$$

then system (1.1) is oscillatory. 
Recently, Baoguo [5] proved above theorem when $q(t)$ is allowed to take on negative values and established the following theorem.

Theorem 1.2. Assume that $\mathbb{T}$ satisfies condition (C) and (1.2). If there exists $\lambda \in[0,1)$ such that

$$
\int_{t_{0}}^{\infty} \bar{p}(\sigma(t)) q(t) \Delta t=\infty,
$$

where $\bar{p}(t)$ is defined by (1.3), then system (1.1) is oscillatory.

To be precise, we say $\mathbb{T}$ satisfies condition (C), that is there is an $M>0$ such that $\chi(t) \leq$ $M \mu(t), t \in \mathbb{T}$, where $\chi$ is the characteristic function of the set $\hat{\mathbb{V}}=\{t \in \mathbb{T}: \mu(t)>0\}$. We note that if $\mathbb{T}$ satisfies condition $(\mathrm{C})$, then the subset $\check{\mathbb{T}}$ of $\mathbb{T}$ defined by

$$
\check{\mathbb{T}}=\{t \in \mathbb{T}: t>0 \text { is right-scattered or left-scattered }\} \text {, }
$$

is necessarily countable and $\hat{\mathbb{T}} \subset \check{\mathbb{T}}$. Then, we can rewrite $\check{\mathbb{T}}$ by

$$
\check{\mathbb{T}}=\left\{t_{i} \in \mathbb{T}: 0<t_{1}<t_{2}<\ldots<t_{n}<\ldots\right\},
$$

and so

$$
\mathbb{T}=\check{\mathbb{T}} \cup\left[\cup_{n \in A}\left(t_{n-1}, t_{n}\right)\right],
$$

where $A$ is the set of all integers for which the real open interval $\left(t_{n-1}, t_{n}\right)$ is contained in $\mathbb{T}$. There are several time scales do not satisfy condition $(C)$, for example It is easy to see the time scale in the form

$$
\mathbb{T}:=\bigcup_{k=1}^{\infty} T_{k} \text {, where } T_{k}=\bigcup_{n=1}^{\infty}\left\{k+\frac{n+1}{n}\right\},
$$

does not satisfy condition (C).

Therefore it will be of great interest to prove Theorems 1.1 and 1.2 without explicit sign assumption on $q$ and also without condition (C) to the same dynamic system (1.1), so our work improves and generalizes those established in $[6,5]$.

\section{Main results}

Before stating the main results, we start with the following lemmas which will play an important role in the proofs of the main results.

Lemma 2.1. For all $\lambda \in[0,1)$ and for sufficiently large $T \geq t_{0}$, we have, for $t \in[T, \infty) \mathbb{T}$

$$
\int_{T}^{t} p(s)[\bar{p}(\sigma(s))]^{\lambda-2} \Delta s \leq \frac{[\bar{p}(T)]^{\lambda-1}}{1-\lambda},
$$

where $\bar{p}(t)$ is defined by (1.3). 
Proof. By Pötzsche chain rule ([3, Theorem 1.90]), we have

$$
\begin{aligned}
\frac{1}{\lambda-1}\left([\bar{p}(s)]^{\lambda-1}\right)^{\Delta} & =\int_{0}^{1}[(1-h) \bar{p}(s)+h \bar{p}(\sigma(s))]^{\lambda-2} d h p(s) \\
& \geq \int_{0}^{1}[(1-h) \bar{p}(\sigma(s))+h \bar{p}(\sigma(s))]^{\lambda-2} d h p(s) \\
& =[\bar{p}(\sigma(s))]^{\lambda-2} p(s) .
\end{aligned}
$$

which yields

$$
\begin{aligned}
\int_{T}^{t} p(s)[\bar{p}(\sigma(s))]^{\lambda-2} \Delta s & \leq \frac{1}{\lambda-1} \int_{T}^{t}\left([\bar{p}(s)]^{\lambda-1}\right)^{\Delta} \Delta s \\
& =\frac{1}{\lambda-1}\left\{[\bar{p}(t)]^{\lambda-1}-[\bar{p}(T)]^{\lambda-1}\right\} \\
& \leq \frac{[\bar{p}(T)]^{\lambda-1}}{1-\lambda} .
\end{aligned}
$$

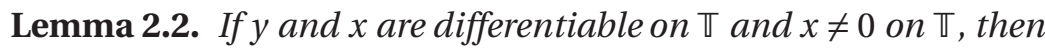

$$
x^{\Delta}\left(\frac{y^{2}}{x}\right)^{\Delta}=\left(y^{\Delta}\right)^{2}-\left[\frac{\frac{x^{\Delta} y^{\sigma}}{x}}{\sqrt{1+\mu \frac{x^{\Delta}}{x}}}+\sqrt{1+\mu \frac{x^{\Delta}}{x}} y^{\Delta}\right]^{2} .
$$

Proof. From the quotient rule, we get

$$
x^{\Delta}\left(\frac{y^{2}}{x}\right)^{\Delta}=x^{\Delta}\left[\frac{x\left(y^{2}\right)^{\Delta}-y^{2} x^{\Delta}}{x x^{\sigma}}\right]=\frac{x^{\Delta}}{x x^{\sigma}}\left[x\left(y^{2}\right)^{\Delta}-y^{2} x^{\Delta}\right]
$$

Since $\left(y^{2}\right)^{\Delta}=(y y)^{\Delta}=y^{\Delta}\left(y^{\sigma}+y\right)=y^{\Delta}\left(2 y+\mu y^{\Delta}\right)$, then

$$
\begin{aligned}
x^{\Delta}\left(\frac{y^{2}}{x}\right)^{\Delta} & =\frac{x^{\Delta}}{x x^{\sigma}}\left[2 x y y^{\Delta}+\mu x\left(y^{\Delta}\right)^{2}-x^{\Delta} y^{2}\right] \\
& =\frac{x^{\Delta}}{x x^{\sigma}}\left[2 y y^{\Delta}\left(x^{\sigma}-\mu x^{\Delta}\right)+\mu\left(y^{\Delta}\right)^{2}\left(x^{\sigma}-\mu x^{\Delta}\right)-x^{\Delta} y^{2}\right] \\
& =\frac{x^{\Delta}}{x x^{\sigma}}\left[-x^{\Delta}\left(y^{2}+2 \mu y y^{\Delta}+\mu^{2}\left(y^{\Delta}\right)^{2}\right)+x^{\sigma} y^{\Delta}\left(y+\mu y^{\Delta}\right)+x^{\sigma} y y^{\Delta}\right] \\
& =\frac{x^{\Delta}}{x x^{\sigma}}\left[-x^{\Delta}\left(y+\mu y^{\Delta}\right)^{2}+x^{\sigma} y^{\Delta}\left(y+\mu y^{\Delta}\right)+x^{\sigma} y^{\Delta}\left(y^{\sigma}-\mu y^{\Delta}\right)\right] \\
& =\frac{x^{\Delta}}{x x^{\sigma}}\left[-x^{\Delta}\left(y^{\sigma}\right)^{2}+2 x^{\sigma} y^{\Delta} y^{\sigma}-\mu x^{\sigma}\left(y^{\Delta}\right)^{2}\right] \\
& =-\left(\frac{x^{\Delta} y^{\sigma}}{x}\right)^{2} \frac{x}{x^{\sigma}}+\frac{2 x^{\Delta}}{x} y^{\Delta} y^{\sigma}-\frac{\mu x^{\Delta}}{x}\left(y^{\Delta}\right)^{2} \\
& \left.=-\frac{x^{\Delta} y^{\sigma}}{x}\right)^{2} \\
1+\mu \frac{x^{\Delta}}{x} & \frac{2 x^{\Delta}}{x} y^{\Delta} y^{\sigma}-\frac{\mu x^{\Delta}}{x}\left(y^{\Delta}\right)^{2}
\end{aligned}
$$




$$
=-\left[\frac{\frac{x^{\Delta} y^{\sigma}}{x}}{\sqrt{1+\mu \frac{x^{\Delta}}{x}}}+\sqrt{1+\mu \frac{x^{\Delta}}{x}} y^{\Delta}\right]^{2}+\left(y^{\Delta}\right)^{2}
$$

since $1+\mu \frac{x^{\Delta}}{x}=\frac{x^{\sigma}}{x} \neq 0$.

From above lemmas and motivated by the proof of Theorem 3.1 in [5], we can prove Theorem 1.1 without condition (C).

Theorem 2.3. Assume that

$$
\int_{t_{0}}^{\infty} p(t) \Delta t=\infty \text { and } \lim _{t \rightarrow \infty} \frac{\mu(t) p(t)}{\bar{p}(t)}=0 .
$$

If there exists $\lambda \in[0,1)$ such that

$$
\int_{t_{0}}^{\infty} \bar{p}^{\lambda}(\sigma(t)) q(t) \Delta t=\infty
$$

where $\bar{p}(t)$ is defined by (1.3), then system (1.1) is oscillatory.

Proof. Assume that $(u(t), v(t))$ is a nonoscillatory solution of system (1.1). We claim that $u(t)$ is nonoscillatory. If not, we assume $u(t)$ is oscillatory and $v(t)$ is nonoscillatory. Without loss of generality, we let $v(t)>0$ on $\left[t_{0}, \infty\right)_{\mathbb{T}}$. In the view of the first equation of system (1.1), we have $u^{\Delta}(t)>0$ on $\left[t_{0}, \infty\right)_{\mathbb{T}}$. Thus $u(t)>0$ or $u(t)<0$ for all large $t$, which is a contradiction. Thus $u(t)$ is nonoscillatory and without loss of generality, we let $u(t)>0$ for $t \geq T \geq t_{0}$. Define, for $\lambda \in[0,1)$

$$
w(t):=\frac{\bar{p}^{\lambda}(t) u^{\Delta}(t)}{p(t) u(t)} .
$$

Then, from the product rule, we get (suppressing arguments)

$$
w^{\Delta}=\left[\frac{\bar{p}^{\lambda}}{u}\right]^{\sigma}\left(\frac{1}{p} u^{\Delta}\right)^{\Delta}+\left[\frac{\bar{p}^{\lambda}}{u}\right]^{\Delta}\left(\frac{1}{p} u^{\Delta}\right)=-\left(\bar{p}^{\lambda}\right)^{\sigma} q+\frac{1}{p}\left[u^{\Delta}\left(\frac{\left(\bar{p}^{\lambda / 2}\right)^{2}}{u}\right)^{\Delta}\right] .
$$

Now, by using Lemma 2.2 with replaced $x$ by $u$ and $y$ by $\bar{p}^{\lambda / 2}$, we get

$$
\begin{aligned}
w^{\Delta} & =-\left(\bar{p}^{\lambda}\right)^{\sigma} q+\frac{1}{p}\left[u^{\Delta}\left(\frac{\left(\bar{p}^{\lambda / 2}\right)^{2}}{u}\right)^{\Delta}\right] \\
& =-\left(\bar{p}^{\lambda}\right)^{\sigma} q+\frac{1}{p}\left[\left(\left(\bar{p}^{\lambda / 2}\right)^{\Delta}\right)^{2}-\left[\frac{\frac{u^{\Delta}\left(\bar{p}^{\lambda / 2}\right)^{\sigma}}{u}}{\sqrt{1+\mu \frac{u^{\Delta}}{u}}}+\sqrt{1+\mu \frac{u^{\Delta}}{u}} \bar{p}^{\lambda / 2 \Delta}\right]^{2}\right]
\end{aligned}
$$




$$
\leq-\left(\bar{p}^{\lambda}\right)^{\sigma} q+\frac{\left(\left(\bar{p}^{\lambda / 2}\right)^{\Delta}\right)^{2}}{p}
$$

Therefore,

$$
\int_{T}^{t} w^{\Delta}(s) \Delta s \leq-\int_{T}^{t} \bar{p}^{\lambda}(\sigma(s)) q(s) \Delta s+\int_{T}^{t} \frac{\left[\left(\bar{p}^{\lambda / 2}(s)\right)^{\Delta}\right]^{2}}{p(s)} \Delta s .
$$

Using the Pötzsche chain rule ([3, Theorem 1.90]), we get

$$
\left(\bar{p}^{\lambda / 2}(t)\right)^{\Delta}=\frac{\lambda}{2} \int_{0}^{1}[(1-h) \bar{p}(t)+h \bar{p}(\sigma(t))]^{\lambda / 2-1} d h p(t) .
$$

Since $\lim _{t \rightarrow \infty} \frac{\mu(t) p(t)}{\bar{p}(t)}=0$, it implies that

$$
\lim _{t \rightarrow \infty} \frac{\bar{p}(\sigma(t))}{\bar{p}(t)}=\lim _{t \rightarrow \infty} \frac{\int_{t_{0}}^{t} p(s) \Delta s+\int_{t}^{\sigma(t)} p(s) \Delta s}{\int_{t_{0}}^{t} p(s) \Delta s}=1+\lim _{t \rightarrow \infty} \frac{\mu(t) p(t)}{\bar{p}(t)}=1,
$$

so for given $0<\epsilon<1$, there exists $T_{1}$ sufficiently large such that

$$
\bar{p}(t) \geq(1-\epsilon) \bar{p}(\sigma(t)), \quad \text { for } t \geq T_{1} .
$$

Then, from (2.4), we have

$$
\begin{aligned}
\left(\bar{p}^{\lambda / 2}(t)\right)^{\Delta} & \leq \frac{\lambda}{2} p(t)[\bar{p}(\sigma(t))]^{\lambda / 2-1} \int_{0}^{1}[1-(1-h) \epsilon]^{\lambda / 2-1} d h \\
& =p(t)[\bar{p}(\sigma(t))]^{\lambda / 2-1} \frac{1}{\epsilon}\left[1-(1-\epsilon)^{\lambda / 2}\right] \\
& =M p(t)[\bar{p}(\sigma(t))]^{\lambda / 2-1},
\end{aligned}
$$

where $M:=\frac{1}{\epsilon}\left[1-(1-\epsilon)^{\lambda / 2}\right]$. From (2.3) and (2.5), we get

$$
\begin{aligned}
w(t)-w(T) & \leq-\int_{T}^{t} \bar{p}^{\lambda}(\sigma(s)) q(s) \Delta s+\int_{T}^{t} \frac{\left[\left(\bar{p}^{\lambda / 2}(s)\right)^{\Delta}\right]^{2}}{p(s)} \Delta s \\
& \leq-\int_{T}^{t} \bar{p}^{\lambda}(\sigma(s)) q(s) \Delta s+M^{2} \int_{T}^{t} p(s)[\bar{p}(\sigma(s))]^{\lambda-2} \Delta s .
\end{aligned}
$$

Since

$$
\begin{aligned}
\frac{1}{\lambda-1}\left([\bar{p}(s)]^{\lambda-1}\right)^{\Delta} & =\int_{0}^{1}[(1-h) \bar{p}(s)+h \bar{p}(\sigma(s))]^{\lambda-2} d h p(s) \\
& \geq \int_{0}^{1}[(1-h) \bar{p}(\sigma(s))+h \bar{p}(\sigma(s))]^{\lambda-2} d h p(s) \\
& =[\bar{p}(s)]^{\lambda-2} p(s),
\end{aligned}
$$


which yields

$$
\begin{aligned}
w(t)-w(T) & \leq-\int_{T}^{t} \bar{p}^{\lambda}(\sigma(s)) q(s) \Delta s+M^{2}\left(\frac{[\bar{p}(t)]^{\lambda-1}}{\lambda-1}-\frac{[\bar{p}(T)]^{\lambda-1}}{\lambda-1}\right) \\
& \leq-\int_{T}^{t} \bar{p}^{\lambda}(\sigma(s)) q(s) \Delta s+M^{2}\left(\frac{[\bar{p}(T)]^{\lambda-1}}{1-\lambda}\right) .
\end{aligned}
$$

In view of condition (2.2), it follows from the last inequality that there exists a sufficiently large $T_{2} \geq T_{1}$ such that

$$
u^{\Delta}(t)<0, \text { for } t \in\left[T_{2}, \infty\right) \mathbb{\pi} .
$$

By using [3, Theorem 4.61], there is a positive solution $\widetilde{u}$, called dominant solution such that

$$
\int_{T_{2}}^{\infty} \frac{p(t)}{\widetilde{u}(t) \widetilde{u}^{\sigma}(t)} \Delta t<\infty,
$$

and then, from (2.6), we get

$$
\widetilde{u}^{\Delta}(t)<0, \quad \text { for } t \in\left[T_{2}, \infty\right) \mathbb{\pi} .
$$

This implies

$$
\begin{aligned}
\int_{T_{2}}^{\infty} p(t) \Delta t & =\widetilde{u}\left(T_{2}\right) \widetilde{u}^{\sigma}\left(T_{2}\right) \int_{T_{2}}^{\infty} \frac{p(t)}{\widetilde{u}\left(T_{2}\right) \widetilde{u}^{\sigma}\left(T_{2}\right)} \Delta t \\
& \leq \widetilde{u}\left(T_{2}\right) \widetilde{u}^{\sigma}\left(T_{2}\right) \int_{T_{2}}^{\infty} \frac{p(t)}{\widetilde{u}(t) \widetilde{u}^{\sigma}(t)} \Delta t<\infty,
\end{aligned}
$$

which is a contradiction. This completes the proof.

\section{References}

[1] D. R. Anderson, Oscillation and nonoscillation criteria for two-dimensional time-scale systems of first-order nonlinear dynamic equations, Electron. J. Diff. Eqns, Vol 2009(2009), No. 24, 1-13.

[2] M. Bohner, L. Erbe, and A. Peterson, Oscillation for nonlinear second order dynamic equations on a time scale, J. Math. Anal. Appl., 301 (2005), 491-507.

[3] M. Bohner and A. Peterson, Dynamic Equation on Time Scales: An Introduction with Applications, Birkhäuser, Boston, 2001.

[4] M. Bohner and A. Peterson, editors, Advances in Dynamic Equations on Time Scales, Birkhäuser, Boston, 2003.

[5] J. Baoguo, A new oscillation criterion for two-dimensional dynamic systems on time scales, Tamkang journal of Mathematics, 42 (2011), 237-244.

[6] S. Fu and M. Lin, Oscillation and nonoscillation criteria for linear dynamic equations on time scales, Computers and Mathematics with Applications, 59 (2010), 2552-2565.

[7] C. Pötzsche, Chain rule and invariance principle on measure chains, in: R. P. Agarwal, M. Bohner, D. O'Regan (Eds.), special Issue on "Dynamic Equations on Time Scales", J. Comput. Appl. Math., 141 (2002), $249-254$.

Department of Mathematics, Faculty of Science, Mansoura University, Mansoura, 35516, Egypt.

Department of Mathematics, Faculty of Science, Hail University, KSA.

E-mail: tshassan@mans.edu.eg 